\title{
Confianza en la mnemotecnia de la palabra clave para aprender lenguas
}

\author{
Confidence in the keyword mnemonics to learn languages
}

\author{
Leticia Ameijide, Alfredo Campos \\ Departamento de Psicología Social, Básica y Metodología, Universidad de Santiago de Compostela, España
}

\begin{abstract}
Resumen
El objetivo principal de esta investigación era averiguar si la mnemotecnia de la palabra clave reforzada con dibujos es eficaz en estudiantes de ESO y, por otro lado, el nivel de confianza de esta estrategia. La muestra estaba compuesta por un grupo de 104 participantes que aprendieron la traducción de una lista corta de 16 palabras latinas. El grupo control aprendió por el método de repetición y el grupo experimental a través de la mnemotecnia de la palabra clave reforzada con dibujos. Los resultados indicaron que el género no influyó en el recuerdo y seguridad de la respuesta. Sin embargo, la estrategia de aprendizaje sí influyó. Palabras clave: aprendizaje, mnemotecnia de la palabra clave, dibujos, nivel de confianza, recuerdo.
\end{abstract}

\begin{abstract}
The main goal of this investigation was to find out if the keyword mnemonics reinforced with drawings is effective for ESO students and, on the other hand, the confidence level of this strategy. The sample was composed by a group of 104 participants who learned the translation of a short list of 16 Latin words. The control group learned by the repetition method and the experimental group learned by the keyword mnemonics reinforced with drawings. The results indicated that gender did not have an influence in the recall and sureness of the response. However, the learning strategy proved to have an influence.

Keywords: learning, keyword mnemonics, drawings, confidence level, recall.
\end{abstract}

El cerebro está dividido en dos partes: el hemisferio izquierdo y el hemisferio derecho. Nuestro sistema educativo está basado únicamente en el aprendizaje a través del hemisferio izquierdo, el cual es analítico, racional y verbal. Para lograr aprendizajes más duraderos, se debe utilizar la imaginación mental, una capacidad del hemisferio derecho. De este modo, es posible mejorar los procesos cognitivos (atención, memoria, pensamiento,...) implicados en el aprendizaje del ser humano. Según la Teoría de la Codificación Dual de Paivio (1975), la información se almacena en la memoria mediante dos códigos: verbal y visual. Para adquirir una mayor comprensión del aprendizaje y que los resultados académicos mejoren, resulta esencial que ambos lados del cerebro actúen de manera equilibrada y armónica.

La regla mnemotécnica más conocida para aprender lenguas extranjeras es la mnemotecnia de la palabra clave o "keyword" (Atkinson, 1975). Este método basado en el principio de asociación consta de dos pasos. En el primero, "verbal", se elabora una palabra clave concreta y con semejanza fonética u ortográfica a la palabra extranjera que se debe aprender. En el segundo paso, "visual", se crea una imagen mental relacionando la palabra clave con el significado de la palabra extranjera (Campos, Amor y González, 2002; Campos, Camino y Pérez-Fabello, 2011; González, Amor y Campos, 2003). Muchas investigaciones han demostrado la eficacia de la mnemotecnia de la palabra clave tanto en niños y jóvenes (Campos, Amor y González, 2004a; Campos, RodríguezPinal y Pérez-Fabello, 2013, 2014) como en personas adultas (Troutt-Ervin, 1990).

Por su parte, Campos y colaboradores (Campos, Amor et al., 2004a; Campos, Amor y González, 2004b; Campos, González y Amor, 2004) propusieron una nueva alternativa en sus trabajos que consistió en que los compañeros de los participantes experimentales (similares en edad, nivel educativo y desarrollo cognitivo) generen la palabra clave. Así, la palabra clave evocada será la que le salga más espontáneamente a los participantes con el fin de mejorar el recuerdo de la traducción española. Con este nuevo método, la mnemotecnia de la palabra clave fue superior a otros métodos de aprendizaje tanto con jóvenes (Campos et al., 2002; Campos, Amor et al., 2004a, 2004b; Campos et al., 2004; Campos et al., 2013, 2014) como con personas adultas (Campos, Pérez-Fabello y Camino, 2010).

Para mejorar el rendimiento en las tareas de memoria existen dos maneras. Una de ellas consiste en reforzar la estrategia de la mnemotecnia de la palabra clave con imágenes mentales (Campos, Amor et al., 2004a). Y la otra, se basa en presentar además un dibujo que relacione la palabra clave con la traducción española de la palabra latina. Campos y Ameijide (2014) comprobaron que el recuerdo inmediato resulta mejor al usar palabras clave con dibujos que sin ellos (Carney y Levin, 2000). Y si nos centramos en la eficacia de la mnemotecnia de la palabra clave generada por compañeros y suscitada con dibujos, se produce un recuerdo mayor que con otro tipo de técnicas de aprendizaje (Amor, Campos y González, 2003; Campos et al., 2002; Campos, Amor et al., 2004b).

No obstante, a la hora de recordar el material que es objeto de aprendizaje, no sólo influyen las estrategias mnemotécnicas sino también la metamemoria (Ameijide 
y Campos, 2011a, 2011b; Campos y Ameijide, 2014), entendida ésta como el conocimiento y el control que posee el individuo sobre el funcionamiento de su memoria, incluyendo sus diferentes fases: codificación, almacenamiento y recuperación (Cosentino, Metcalfe, Holmes, Steffener y Stern, 2011; Metcalfe, Eich y Castel, 2010).

Dentro del modelo general de memoria, Nelson y Narens (1990) plantean el estudio de la metamemoria en tres etapas principales: adquisición (antes del aprendizaje propiamente dicho), retención (mantenimiento del conocimiento previamente adquirido) y recuperación consciente de la información. Los juicios metamnemónicos se pueden llevar a cabo en cuatro momentos del proceso de aprendizaje: a) antes del proceso de adquisición (juicios de facilidad de aprendizaje, EOL = ease of learning judgments), b) durante o inmediatamente después de la adquisición, pero antes de la prueba de recuperación (juicios de aprendizaje, JOL = judgments of learning) y c) después de la recuperación del material durante la prueba (juicios de confianza, $\mathrm{CL}=$ confidence level judgments). Existen pocos trabajos en los que se analizó la influencia de la mnemotecnia de la palabra clave sobre los juicios metamnemónicos (Ameijide y Campos, 2011a, 2011b; Campos y Ameijide, 2011, 2014). Por ello, el objetivo principal de esta investigación era averiguar si la mnemotecnia de la palabra clave reforzada con dibujos es eficaz en estudiantes de Educación Secundaria Obligatoria (ESO) y, por otro lado, el nivel de confianza de esta estrategia.

\section{Método}

\section{Participantes}

La muestra estaba compuesta por un grupo de 104 participantes (58 hombres y 46 mujeres) con una media de edad de 15.25 años $(S D=.75)$. Los participantes eran estudiantes del Instituto de Educación Secundaria Plurilingüe Eusebio da Guarda de A Coruña que pertenecían a $3^{\circ}$ y $4^{\circ}$ curso de Educación Secundaria Obligatoria (ESO). Todos participaron voluntariamente.

\section{Instrumentos}

Se empleó una lista corta de 16 palabras latinas ( 8 altas en viveza de imagen y 8 bajas en viveza de imagen), con sus respectivas palabras clave, las traducciones españolas de las palabras latinas, las frases interactivas entre cada palabra clave y el significado de la palabra latina y finalmente, 16 dibujos que representaban el significado de dichas frases interactivas. Para el entrenamiento se utilizaron 4 palabras latinas ( 2 altas en viveza de imagen y 2 bajas en viveza de imagen).

\section{Procedimiento}

En primer lugar, se seleccionaron de manera aleatoria un conjunto de 24 palabras latinas, 12 altas en viveza de imagen y 12 bajas en viveza de imagen, de las publicadas por Valle (1998). A continuación, se consideraron palabras altas en viveza de imagen las que tenían una puntuación superior a 5.20 y palabras bajas en viveza de imagen las que tenían una puntuación inferior a 4.21. Estas puntuaciones se obtuvieron al sumar y restar a la media, 2/5 de la desviación típica, según el estudio de investigación de Valle (1998). Posteriormente, se tradujeron las palabras al latín y se rechazaron aquellos vocablos que sonasen o se escribiesen iguales o muy parecidos en los dos idiomas (latín y español). El motivo principal de utilizar como idioma el latín era porque los participantes lo desconocían y por consiguiente, partían del mismo nivel de conocimientos.

A continuación, esta lista de 24 palabras latinas se le presentó a un grupo de 15 participantes elegidos al azar, de la misma edad y características socioculturales que los participantes experimentales para que, sin límite de tiempo, elaborasen las palabras clave (palabras españolas), lo más concretas posible y que sonasen o se escribiesen lo más parecido posible a las palabras latinas. Después, debían crear una frase para cada palabra en la que aparecía reflejada la relación de interacción entre la palabra clave y la traducción española de la palabra latina. Posteriormente, los investigadores seleccionaron las palabras clave de mayor frecuencia de aparición y las frases interactivas más repetidas. Por ejemplo, palabra latina "Delineatio", palabra clave "Delineante", traducción española "Esquema" y frase interactiva "El delineante está haciendo un esquema". Finalmente, se rechazaron las palabras clave menos frecuentes hasta quedar con una lista definitiva de 16 palabras latinas ( 8 altas en viveza de imagen y 8 bajas en viveza de imagen) que fueron las que se presentaron a los participantes como objetivo de aprendizaje en el transcurso del experimento. La media de imagen de las palabras altas en imagen fue de $6.27(S D=.35)$ y la media de las palabras bajas en imagen fue de $3.55(S D=.54)$. La media en viveza de imagen del total de las palabras fue de 4.91 $(S D=1.47)$. La diferencia en viveza de imagen entre las palabras altas en imagen y las bajas en imagen fue significativa, $t(1,14)=-11.91, p<.001)$. También se seleccionaron 4 palabras latinas ( 2 altas en viveza de imagen y 2 bajas en viveza de imagen) para el entrenamiento.

Los 104 participantes fueron distribuidos aleatoriamente en dos grupos. El grupo control formado por 48 participantes ( 26 hombres y 22 mujeres) tenía la tarea de aprender el significado español de 16 palabras latinas utilizando el método de repetición. Esta lista corta de 16 palabras latinas se les presentó en diapositivas a través de un proyector digital conectado a un ordenador. En cada diapositiva aparecía la palabra latina y la traducción española y un experimentador pronunciaba dos veces, en voz alta, la palabra latina y la traducción española para que el participante las aprendiese utilizando el método de repetición. El grupo experimental compuesto por 56 participantes (32 hombres y 24 mujeres) aprendió la misma lista de palabras mediante la mnemotecnia de la palabra clave reforzada con dibujos, siguiendo el mismo procedimiento que el grupo control, excepto que en las diapositivas del grupo experimental aparecía la palabra latina, la palabra clave, la traducción española de la palabra latina, una frase que representaba la palabra clave interactuando con el significado de la palabra latina y un dibujo reflejando esa interacción. Un experimentador leía dos veces las tres palabras y la frase interactiva para que el participante formase una imagen 
mental entre la palabra clave y el significado español de la palabra latina. El ritmo de presentación de cada diapositiva fue de 15 segundos para los dos grupos.

Una vez que a los participantes se les presentaron las 4 palabras latinas de entrenamiento, éstos tenían que escribir en un folio, al lado de cada palabra latina, su correspondiente traducción española. Posteriormente, se mostró la lista corta de 16 palabras latinas para el aprendizaje a través de un proyector digital de diapositivas conectado a un ordenador. En otro folio, se les presentó la lista corta de 16 palabras latinas para que cada participante escribiera al lado de cada palabra en latín, la respectiva traducción española con el objetivo de medir el recuerdo de los estudiantes. Finalmente, en una escala de 1 a 5 , tenían que puntuar el nivel de confianza (CL) o de seguridad de que dicha respuesta que habían escrito era la correcta. El 1 indicaba "estoy completamente seguro de que la respuesta es incorrecta", y el 5 indicaba "estoy completamente seguro de que la respuesta es correcta". A los participantes se les dio un tiempo total de 3 minutos para realizar ambas tareas. Una vez que cada participante contestó el recuerdo y el juicio de confianza (CL) finalizó el experimento.

\section{Resultados}

Para averiguar si el género (hombre y mujer) y la estrategia de aprendizaje (método de repetición y mnemotecnia de la palabra clave reforzada con dibujos) influyeron en el recuerdo y en la seguridad de la respuesta de los participantes se llevaron a cabo dos Análisis Univariados de Varianza (ANOVA). Las medias y desviaciones típicas obtenidas en el recuerdo y en la seguridad de la respuesta se encuentran en la Tabla 1.

Tabla 1.

Medias y Desviaciones Típicas del Recuerdo y de la Seguridad de la Respuesta.

\begin{tabular}{|c|c|c|c|c|c|c|c|c|c|c|}
\hline & \multicolumn{4}{|c|}{ Estrategia } & \multicolumn{4}{|c|}{ Género } & & \\
\hline & \multicolumn{2}{|c|}{ P. Clave } & \multicolumn{2}{|c|}{ Repetición } & \multicolumn{2}{|c|}{ Mujeres } & \multicolumn{2}{|c|}{ Hombres } & \multicolumn{2}{|c|}{ Total } \\
\hline & $M$ & $S D$ & $M$ & $S D$ & $M$ & $S D$ & $M$ & $S D$ & $M$ & $S D$ \\
\hline Recuerdo & 13.29 & 3.08 & 8.75 & 3.78 & 11.60 & 3.74 & 10.88 & 4.35 & 11.19 & 4.09 \\
\hline Seguridad & 68.54 & 12.62 & 53.40 & 12.55 & 62.00 & 14.98 & 61.20 & 14.49 & 61.55 & 14.64 \\
\hline
\end{tabular}

En primer lugar, se comprobó si el género (hombre y mujer) influyó a la hora de recordar el material objeto de aprendizaje. Los resultados indicaron que el género (hombre y mujer) no influyó significativamente en el recuerdo, $F(1,100)=1.67, p=.20$. Sin embargo, se encontró que la estrategia de aprendizaje (método de repetición y mnemotecnia de la palabra clave reforzada con dibujos) tuvo una influencia significativa en el recuerdo, $F(1,100)=42.70, p<.001$. Por tanto, los participantes que emplearon la mnemotecnia de la palabra clave reforzada con dibujos $(M=13.29$, $S D=3.08$ ) tuvieron un mayor recuerdo que los individuos que aprendieron con el método de repetición $(M=8.75, S D=3.78)$. Además, la interacción entre las dos variables (género y estrategia de aprendizaje) fue significativa, $F(1,100)=4.16, p=.04$.

En segundo lugar, se analizó si existían diferencias significativas tanto en el género (hombre y mujer) como en la estrategia de aprendizaje (método de repetición y mnemotecnia de la palabra clave reforzada con dibujos) a la hora de que los participantes mostraran su nivel de confianza en sus respuestas. Se encontró que el género (hombre y mujer) no influyó de manera significativa en la seguridad de sus respuestas, $F(1,100)=.21, p=.65$; mientras que la estrategia de aprendizaje (método de repetición y mnemotecnia de la palabra clave reforzada con dibujos) sí resultó significativa, $F(1,100)=34.72, p$ $<$.001. Los estudiantes que aprendieron mediante la mnemotecnia de la palabra clave reforzada con dibujos $(M=68.54, S D=12.62)$ consiguieron un mayor nivel de confianza en sus respuestas que los participantes que utilizaron el método de repetición $(M=53.40$, $S D=12.55)$. Sin embargo, no existió interacción significativa entre las dos variables (género y estrategia de aprendizaje), $F(1,100)=1.40, p=.24$.

\section{Discusión de resultados}

En esta investigación se estudió la influencia del género y la estrategia de aprendizaje tanto en el recuerdo como en la seguridad de la respuesta cuando los estudiantes aprendían vocabulario en un segundo idioma, en este caso el latín.

Los resultados indicaron que el género (hombre y mujer) no tuvo una influencia significativa en el recuerdo ni en la seguridad de la respuesta. Este hallazgo no coincide con el obtenido por Ameijide y Campos (2011a), en el que se demostró que las mujeres obtenían puntuaciones más altas que los hombres en el recuerdo de las palabras. Sin embargo, los hombres fueron los que se mostraron más seguros de sus respuestas una vez dadas.

Atendiendo a la estrategia de aprendizaje (método de repetición y mnemotecnia de la palabra clave reforzada con dibujos) se observó que ambas variables influyeron significativamente. Los participantes que emplearon la mnemotecnia de la palabra clave reforzada con dibujos tuvieron un mayor recuerdo que los individuos que aprendieron con el método de repetición. Este resultado está en la línea de las investigaciones precedentes en las que se comprobó que con la mnemotecnia de la palabra clave generada por compañeros y en ocasiones, también suscitada con dibujos, se produce un mejor recuerdo que 
con otras técnicas de aprendizaje tanto con jóvenes (Amor et al., 2003; Campos et al., 2002; Campos, Amor et al., 2004b; Campos et al., 2004) como con personas mayores (Campos y Ameijide, 2014; Campos et al., 2011; Campos et al., 2010). Además, los participantes lograron un mayor nivel de confianza en sus respuestas con la mnemotecnia de la palabra clave reforzada con dibujos que con el método de repetición. En su investigación con personas mayores, Ameijide y Campos (2011a, 2011b) estuvieron de acuerdo con estos resultados en el sentido de que los participantes que emplearon la mnemotecnia de la palabra clave obtuvieron una mayor seguridad en sus respuestas. En conclusión, esta investigación demuestra la importancia de utilizar las imágenes mentales en el aprendizaje a lo largo de todas las etapas del ciclo vital.

\section{Referencias}

Ameijide, L., y Campos, A. (2011a). Diferencias individuales en la metamemoria cuando se utiliza la mnemotecnia de la palabra clave. Actas del XI Congreso Internacional Galego-Portugués de Psicopedagoxía (pp. 4191-4201). A Coruña: Universidad de A Coruña.

Ameijide, L. y Campos, A. (2011b). Juicios metamnemónicos sobre la mnemotecnia de la palabra clave: estudio con personas mayores. Actas del XI Congreso Internacional Galego-Portugués de Psicopedagoxía (pp. 4203-4211). A Coruña: Universidad de A Coruña.

Amor, A., Campos, A., y González, M. A. (2003). Estilo cognitivo e imagen, ¿afectan a la mnemotecnia de la palabra clave? Revista Galego-Portuguesa de Psicoloxía e Educación, 10, 997-1006.

Atkinson, R. C. (1975). Mnemotechnics in secondlanguage learning. American Psychologist, 30, 821828.

Campos, A., y Ameijide, L. (2011). Exactitud de los juicios de aprendizaje (JOLs) y mnemotecnia de la palabra clave en adultos y ancianos. International Journal of Psychological Research, 4, 64-71.

Campos, A., y Ameijide, L. (2014). Mnemotecnia de la palabra clave con dibujos y juicios metamnemónicos de personas mayores. Revista Iberoamericana de Psicología y Salud, 5, 23-38.

Campos, A., Amor, A., y González, M. A. (2002). Presentation of keywords by means of interactive drawings. The Spanish Journal of Psychology, 5, 102109.

Campos, A., Amor, A., y González, M. A. (2004a). The importance of the keyword- generation method in keyword mnemonics, Experimental Psychology, 51, 125-131.

Campos, A., Amor, A., y González, M. A. (2004b). Drawing-assisted strategies in keyword mnemonics. Studia Psychologica, 46, 211-218.
Campos, A., Camino, E., y Pérez-Fabello, M. J. (2011). Using the keyword mnemonics method among adult learners. Educational Gerontology, 37, 327-335.

Campos, A., González, M. A., y Amor, A. (2004). Different strategies for keyword generation. Journal of Mental Imagery, 28, 51-58.

Campos, A., Pérez-Fabello, M. J., y Camino, E. (2010). Eficacia de la mnemotecnia de la palabra clave en personas adultas. Psicothema, 22, 752-757.

Campos, A., Rodríguez-Pinal, M. D., y Pérez-Fabello, M. J. (2013). Aprendizaje del idioma gallego mediante la mnemotecnia de la palabra clave, en personas bilingües, altas y bajas en control de imagen. Revista de Investigación en Educación, 11, 50-59.

Campos, A., Rodríguez-Pinal, M. D., y Pérez-Fabello, M. J. (2014). Receptive and productive recall with the keyword mnemonics in bilingual students. Current Psychology, 33, 64-72.

Carney, R. N., y Levin, J. R. (2000). Fading mnemonic memories: Here's looking anew, again! Contemporary Educational Psychology, 25, 499-508.

Cosentino, S., Metcalfe, J., Holmes, J., Steffener, J., y Stern, Y. (2011). Finding the self in metacognitive evaluations: Metamemory and agency in non demented elders. Neuropsychology, 25, 602-612.

González, M. A., Amor, A., y Campos, A. (2003). La mnemotecnia de la palabra clave. A Coruña: Servicio de Publicaciones de la Universidad de A Coruña.

Metcalfe, J., Eich, T. S., y Castel, A. D. (2010). Metacognition of agency across the lifespan. Cognition, 116, 267-282.

Nelson, T. O., y Narens, L. (1990). Metamemory: A theoretical framework and new findings. En G. H. Bower (Ed.), The psychology of learning and motivation: Advances in research and theory (pp. 125173). New York, NY: Academic Press.

Paivio, A. (1975). Imagery and long-term memory. En A. Kennedy y A. Wilkes (Eds.), Studies in long term memory (pp. 57-88). London: Wiley.

Troutt-Ervin, E. D. (1990). Application of keyword mnemonics to learning terminology in the college classroom. Journal of Experimental Education, 59, 3141.

Valle, F. (1998). Normas de imaginabilidad. Oviedo: Servicio de Publicaciones de la Universidad de Oviedo.

\section{Agradecimientos}

Quisiera expresar mi agradecimiento al equipo directivo y profesorado del Instituto de Educación Secundaria Plurilingüe Eusebio da Guarda de A Coruña por su colaboración y finalmente, a los alumnos/as de $3^{\circ}$ y $4^{\circ}$ curso de ESO por su participación voluntaria en este estudio de investigación. 